\title{
3D Morphometric Evaluation of Craniofacial Features in Adult Subjects with Marfan Syndrome
}

\author{
Claudia DOLCI ${ }^{1}$, Valentina PUCCIARELLI ${ }^{1}$, Marina CODARI ${ }^{1}$, Susan MARELLI ${ }^{2}$, \\ Giuliana TRIFIRÒ ${ }^{3}$, Alessandro PINI ${ }^{2}$, Chiarella SFORZA ${ }^{* 1}$ \\ ${ }^{1}$ Dipartimento di Scienze Biomediche per la Salute, Università degli Studi di Milano, Milano, Italy; \\ ${ }^{2}$ Centro Malattie Rare, MarfanClinic, ASST Fatebenefratelli-Sacco, Milano, Italy; \\ ${ }^{3}$ U.O. Pediatria, ASST Rhodense, Rho (Milano), Italy \\ DOI: $10.15221 / 16.098 \quad$ http://dx.doi.org/10.15221/16.098
}

\begin{abstract}
Marfan syndrome (MFS) is a hereditable disorder of the connective tissue with an estimated worldwide prevalence of 1:5000, mainly caused by alterations of the extracellular matrix protein fibrillin-1. Cardiovascular manifestations of MFS, including aortic dilation with increased risk of dissection and rupture, are the leading cause of mortality. Therefore, an early and accurate diagnosis of MFS is crucial to prevent the development of complications, even if it may be difficult, due to the variable expression of the disease. It has been suggested that craniofacial abnormalities associated with MFS could predispose to obstructive sleep apnea, which in turn may promote aortic dilation. Since in a previous preliminary study on young subjects with MFS we pointed out some quantitative facial features never described before, we extended the study on adult patients, in order to better characterize the facial phenotype associated with MFS and verify the usefulness of a 3D not invasive quantitative approach for its early recognition. 3D facial images of 49 Italian subjects diagnosed with MFS without previous history of facial injuries or surgery, aged 18-60 years (18 males, mean \pm SD age $37 \pm 11$ years; 31 females, mean \pm SD age $40 \pm 11$ years), and divided in 5 non-overlapping age groups, were obtained by stereophotogrammetry. From the coordinates of 50 soft-tissue facial landmarks, linear distances and angles were measured; $z$ score values were calculated comparing patients with healthy Italian reference subjects (332 males and 329 females), matched for gender and age group. Almost all subjects with MFS (96\%) showed a shorter mandibular ramus than controls (mean z score $=-1.8$ ) and $100 \%$ of subjects showed a greater facial divergence (mean z score $=+2.0$ ), thus strengthening the findings of the previous study on young subjects with MFS. Furthermore, $98 \%$ of subjects showed a reduced ratio between posterior and anterior facial height (mean z score $=-1.8$ ) and $92 \%$ of subjects showed a reduced ratio between facial width and facial height (mean z score = -1.5), being both ratios influenced by an expected but overall mild increase of facial height. Remarkable gender differences or age-specific trends were not observed. Facial abnormalities pointed out in the current 3D morphometric study could represent traits of the phenotypic expression of MFS. Furthermore, since they are in accordance with the findings of our previous study on young patients, their detection could facilitate the early diagnosis of the disease, when the phenotype of MFS is not clearly evident or has not yet been clearly expressed. Further studies on more patients are needed to confirm the promising findings.
\end{abstract}

Keywords: Stereophotogrammetry, Marfan syndrome (MFS), facial morphology

\section{Introduction}

Marfan syndrome (MFS, OMIM \#154700) is a hereditable connective tissue disorder affecting approximately 1 in 5000 people worldwide [1,2]. MFS is due to a mutation in FBN1 (fibrillin-1) gene in more than $95 \%$ of cases. It results in an altered assembly of extracellular matrix fibrils, which in turn leads to a signalling dysregulation of the transforming growth factor beta (TGF- $\beta$ ) family. In the remaining cases, the syndrome is due mostly to mutations in TGF- $\beta$ receptor genes 1 and 2 , and to other still unknown causative genes [3]. MFS is characterized by a broad spectrum of clinical manifestations, involving the musculoskeletal, ocular, cardiovascular, pulmonary, integumental, and nervous systems, with a variable and evolutive phenotypic expression. The most serious complications of MFS result from the pathologic changes in the cardiovascular system. In particular the aorta can weaken and stretch with an increasing risk of dissection [4].

MFS was firstly reported in 1896 by Bernard-Jean Antoine Marfan, who described skeletal alterations including craniofacial features in a 5-year-old girl. Despite the disorder has been known for a long time, to date establishing the diagnosis of MFS can be difficult, also for the current incomplete sensitivity of

\footnotetext{
* chiarella.sforza@unimi.it; +39 02503 15387; www.scibis.unimi.it
} 
molecular genetic testing of FBN1. At present, diagnosis of MFS is based mainly on clinical signs and familiar history, according the revised Ghent nosology [5]. On the other hand, the early suspicion of the disease and a timely and accurate recognition of the disorder is essential to identify patients at risk for an increased morbidity and mortality and improve their life expectancy with appropriate measures [6]. Craniofacial abnormalities associated with MFS may include dolichocephaly, enophthalmos, down-slanting palpebral fissures, malar hypoplasia, and retrognathia. These features are included among the current criteria applied for the diagnosis of MFS, without a clear quantitative definition. They are usually evaluated through a clinical inspection or cranial X-ray examination [7-9]. Besides, most subjects with MFS refer for orthodontic treatment because of narrow jaws and high-arched palate, which can lead to teeth crowding, posterior crossbite and malocclusion [10-15].

It has been suggested that craniofacial abnormalities and increased pharyngeal collapsibility due to abnormal connective tissue associated with MFS could predispose to an increased prevalence of obstructive sleep apnea, which in turn may be a risk factor for aortic dilatation [16,17]. The recognition of dysmorphic facial traits in MFS may improve the early diagnosis of the disease, since the sign of MFS are variable and modify with time, and somatic alterations are usually the first to occur in affected patients [18].

Among different instruments allowing an accurate both qualitative and quantitative assessment of 3D craniofacial morphology [19], stereophotogrammetry is not invasive and significantly improve the cost-benefit ratio of clinical analyses [20]. The morphometric facial analysis through stereophotogrammetry may highlight alterations of facial parameters which may be not detected by an impressive clinical examination of the patients.

In a preliminary study on 11 young subjects with MFS we pointed out some quantitative facial features never described before, alongside with other well known alterations. Noteworthy, subjects showed a longer face than controls, mainly due to an increased middle third, a longer mandibular body with a shorter ramus, and a greater facial divergence [21].

In the current study we extended the investigation on adult patients, in order to better characterize the facial phenotype associated with MFS and verify the usefulness of a 3D not invasive quantitative approach for its early recognition.

\section{Method}

\subsection{Patients and control subjects}

Forty-nine adult subjects diagnosed with MFS according to revised Ghent criteria, referred by the Rare Diseases Center - MarfanClinic - Milan, were recruited for the study. Patients were aged 18-60 years (18 males, mean \pm SD age $37 \pm 11$ years; 31 females, mean \pm SD age $40 \pm 11$ years). They were chosen among white Italian subjects without previous history of craniofacial surgery or trauma and they were divided in 5 non-overlapping groups according to age.

We also examined 661 healthy subjects from the local population (332 males and 329 females), matched with patients for gender, age, and ethnicity, to serve as controls.

All participants provided their informed consent to the investigation, which was preventively approved by the local ethic committee.

\subsection{Data collection and analysis}

For each subject, data collection procedure took place in three steps and it was followed by off-line calculations.

In the first step, an experienced operator identified a set of 50 soft-tissue facial anthropometric landmarks through inspection or palpation, according to international criteria and an experimental protocol developed and widely used in our laboratory to investigate facial features also associated to genetic syndromes [22-30]. Landmarks were marked using a common black eyeliner on the cutaneous surface; during the procedure, which took about 20 minutes, subjects sat relaxed with a natural head position. The complete set of landmarks (Figure 1) allows the quantitative study of head, face, orbits, nose, mouth, and ears in the living human subjects.

In the second step, the three-dimensional facial image was obtained using a stereophotogrammetric system (VECTRA M3, Canfield Scientific Inc, Fairfield, NJ, USA). The instrument has a $1.2 \mathrm{~mm}$ geometry resolution and can reproduce three-dimensional facial morphology with a capture time of 3.5 milliseconds.

In the third step, landmark digitization was performed on 3D facial reconstruction using the marks made on the subjects' skin before each acquisition. 


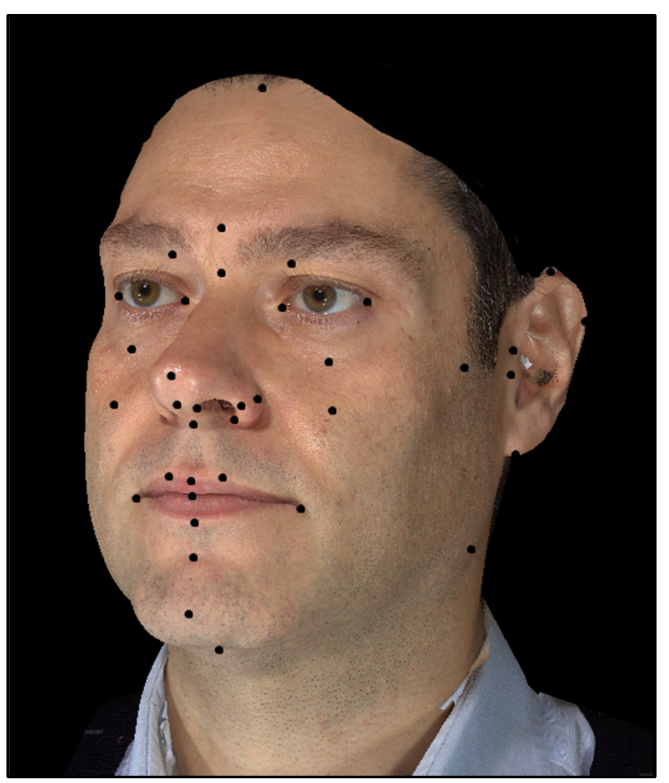

Fig. 1. Fifty facial soft tissue landmarks identified and digitized on subjects with MFS and controls.

Data elaboration was performed off-line and was based on the three-dimensional coordinates of the digitized anthropometric landmarks, which allowed to obtain a geometric model of the face.

The accuracy and reproducibility of landmark identification and marking, and data collection procedures were previously tested and found to be satisfactory [31].

On the basis of the preliminary results of the previous study on young subjects with MFS, from the standard set of 50 landmarks a subset of 3 selected midline landmarks and 2 paired landmarks, were specifically considered in the current study (Table 1). They were used to calculate linear distances, angles and ratios in all three spatial directions by custom computer programs.

Table 1. Subset of facial landmarks used in the current study, and relevant definitions.

\begin{tabular}{lll}
\hline \multicolumn{3}{l}{ Midline landmarks } \\
\hline $\mathrm{n}$ & nasion & at the suture between forehead and nose \\
$\mathrm{sn}$ & subnasale & $\begin{array}{l}\text { at the end of the columella } \\
\text { the most anterior point of the chin }\end{array}$ \\
$\mathrm{pg}$ & pogonion & \\
\hline Paired landmarks & \\
\hline $\mathrm{t}$ & tragion & in the middle of the tragus \\
go & gonion & most lateral point of the mandibular angle \\
\hline
\end{tabular}

Measurements of each patient were transformed in z scores. Calculations were performed using values of the control group of the same gender and corresponding age. Z scores were obtained by subtracting for each patient value its corresponding data from control subject mean value, and dividing by the relevant standard deviation.

Mean z scores and their standard deviations were then calculated, both separately for gender and for age groups, and for the group of patients as a whole. We considered remarkable those mean patient $z$ scores that differed at least 1.5 SD from the control values, since by definition the average $z$ score of control groups is $=0$ and its standard deviation is $=1$.

\section{Results}

Figure 2 shows a representative 3D facial image of a subject with MFS. Anthropometric measurements with remarkable differences in comparison to healthy subjects are highlighted.

Table 2 reports facial anthropometric measurements of adult subjects with MFS with remarkable mean $z$ scores. When compared to control subjects of the same gender and comparable age, almost all subjects with MFS (96\%) showed a shorter mandibular ramus than controls, but a similar mandibular body length, and $100 \%$ of subjects showed a greater facial divergence. 
Furthermore, $98 \%$ of subjects showed a reduced ratio between posterior and anterior facial height and $92 \%$ of subjects showed a reduced ratio between facial width and facial height, being both ratios influenced by an expected but overall mild increase of facial height, mainly due to an increase of its middle third.

Remarkable gender differences or age-specific trends were not observed.

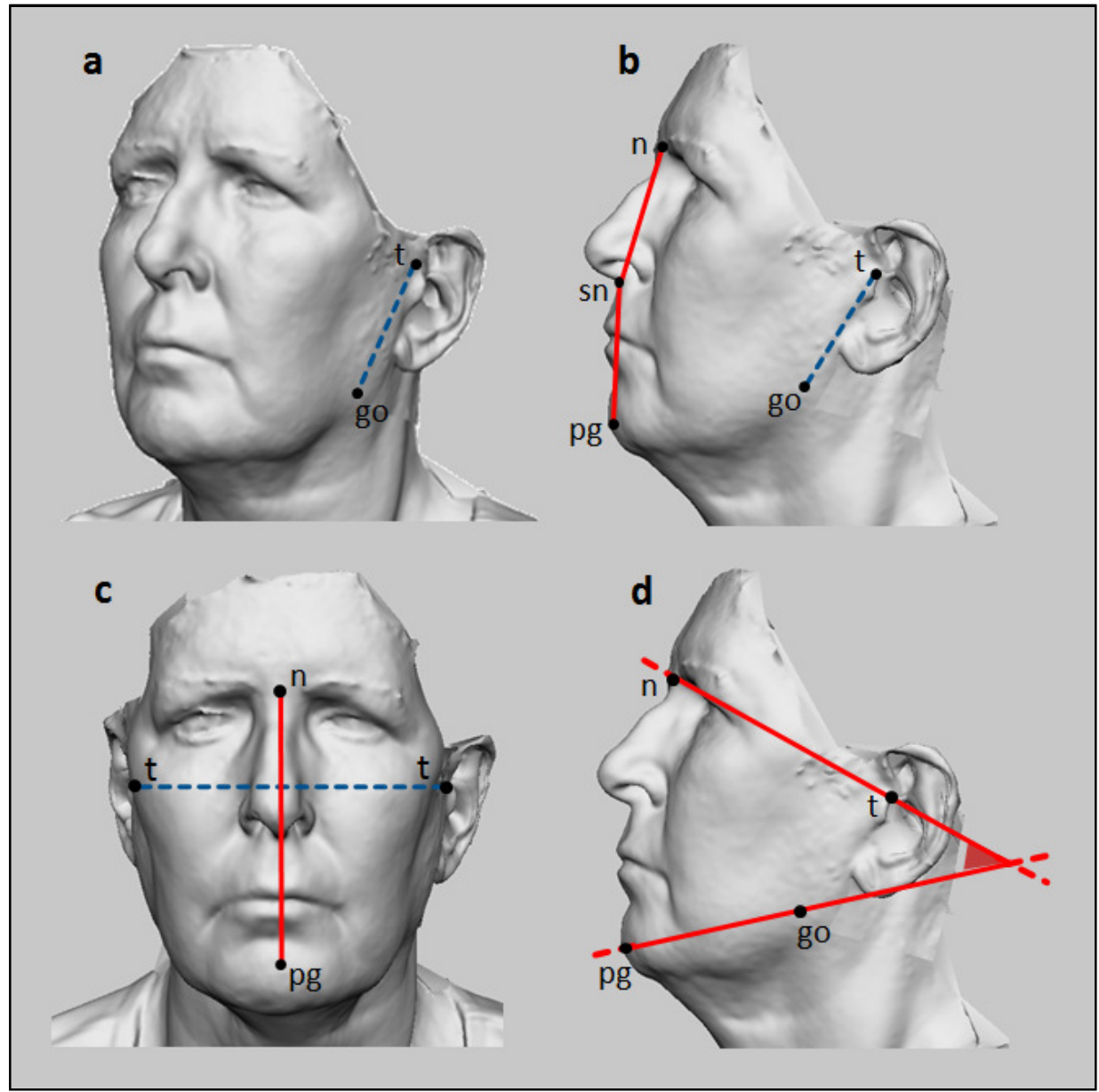

Fig. 2. Representative 3D facial image of an adult subject with MFS. Blue/dotted lines: decreased measurements in comparison to controls.

Red/continue lines and angle: increased measurements in comparison to controls. $t$-go = mandibular ramus length; $n$-sn = length of the middle third of the face; sn-pg =length of the lower third of the face; $n-p g=$ facial height; $t_{r}-t_{l}=$ skull base width; $\left(n-t_{m}\right)-\left(p g-g o_{m}\right)=$ facial divergence.

Table 2. Facial anthropometric measurements in adult subjects with MFS with remarkable mean $z$ score when compared to healthy subjects

\begin{tabular}{llcc}
\hline Measurement & Definition & Mean z score & SD \\
\hline Linear distances & & & \\
$\mathrm{t}_{\mathrm{m}}-\mathrm{go}_{\mathrm{m}}$ & mandibular ramus length & -1.9 & 1.2 \\
$\mathrm{n}$-pg & facial height & +1.3 & 0.9 \\
Angles & & & \\
$\left(\mathrm{n}-\mathrm{t}_{\mathrm{m}}\right)-\left(\mathrm{pg}-\mathrm{go}_{\mathrm{m}}\right)$ & facial divergence & +2.0 & 0.9 \\
Ratios & & & \\
$\left(\mathrm{t}_{\mathrm{m}}-\mathrm{go}_{\mathrm{m}}\right) /(\mathrm{sn}-\mathrm{pg})$ & $\begin{array}{l}\text { posterior/anterior facial height } \\
\left(\mathrm{t}_{\mathrm{r}} \mathrm{t}\right) /(\mathrm{n}-\mathrm{pg})\end{array}$ & -1.9 & 0.8 \\
\hline $\mathrm{r}=$ right, $\mathrm{l}=\mathrm{left}, \mathrm{m}=$ mid-landmark & -1.5 & 1.0 \\
\hline
\end{tabular}

$\mathrm{r}=$ right, $\mathrm{I}=$ left, $\mathrm{m}=$ mid-landmark 


\section{Discussion}

Clinical manifestation of MFS can appear at any age. They may be firstly observed at birth, as well as during childhood or even in adulthood. Nevertheless, people are born with MFS and an early recognition of the disorder allows to prevent its most serious cardiovascular complications, thus allowing for the improvement of life expectancy of the patients. Skeletal abormalities including craniofacial features are often the first sign of the disorder.

Stheneur et al. recently investigated the phenotype evolution during childhood in MFS. Among the skeletal features used for the diagnosis of MFS they did not consider craniofacial features of the patients [32]. Sheikhzadeh et al. developed a clinical model to estimate the probability of MFS, assessing 14 clinical features listed in the Ghent nosology for the diagnosis of the disease. They chose not to include facial features, because clear-cut and reproducible criteria for these characteristics were not available or their assessment required specialized clinical expertise [6]. A study by Ting et al. based on the examination of 2D facial photographs of subjects with MFS concluded that facial features do not have a high sensitivity for MFS and they can be used as an initial screening tool [33].

Few studies investigated quantitative craniofacial features associated with MFS $[7,8,15]$. Actually, direct anthropometry is not routinely used in clinical practice and the assessment of facial dysmorphism in MFS is usually performed through a clinical impression or cranial X-ray examination. Nevertheless, Lipscomb et al. described the classical craniofacial features associated with MFS even before the age of 3-4 years [18], suggesting their potential role for the early diagnosis of the syndrome. The development of different techniques for the three-dimensional reproduction of facial surface morphology allowed the improvement of facial abnormalities recognition, by providing a lot of facial anatomical details. Of particular interest, stereophotogrammetry does not require a physical contact between the instrument and the face thus improving measurement precision, and lacks of motion artifacts because of the very short imaging time. Unlike x-ray examinations, sterophotogrammetry systems do not use ionizing radiation and the facial acquisition is harmless for the subjects. Moreover, they are simple to use and with minimum running costs.

In a preliminary study on young subjects with MFS performed using stereophotogrammetry, we observed that patients had a longer face than controls, mainly due to an increased middle third, a longer mandibular body with a shorter ramus, and a greater facial divergence [21]. In the current study we extended the investigation on adult patients with MFS, in order to verify the previous findings and better characterize the facial phenotype associated with MFS.

Subjects investigated in the present study had a large age range; they were divided in non-overlapping groups according to age, as well as by gender. Due to the limited number of patients for each group, we performed the analysis of facial features using z-scores, which are widely used in anthropometry to assess single patients.

When compared to control subjects, almost all adult subjects with MFS showed a shorter mandibular ramus and an increased facial divergence, like the young patients investigated in the previous study [21]. The second feature was also reported by both Cistulli et al. [7] and De Coster et al. [8] in their cephalometric studies. The two anthropometric measurements could represent a phenotypic trait of MFS. Since they were observed also in young patients, their detection could facilitate the early recognition of the disease, particularly when the phenotype of MFS is not clearly evident or has not yet been clearly expressed, in order to start an effective management and follow up of the disease as soon as possible.

The increase of the mandibular body length described in young patients with MFS was not observed in adult patients. The small number of subjects investigated in the previous study, and the different age range of the analyzed subjects could partly explain the difference. In contrast, the cephalometric study by De Coster et al. reported a short mandibular body [8]. The use of 2D projections could partly explain the difference. Indeed, further investigations are necessary to evaluate the potential role of the mandibular body length in MFS recognition.

In the current study, patients showed a reduced ratio between posterior and anterior facial height and a reduced ratio between facial width and facial height. These findings are in a good agreement with the decrease of the mandibular ramus, the increase of the facial height, in addition to the increase of the facial width observed in the analyzed subjects.

Findings of the current study confirm the usefulness of a new approach in the evaluation of facial dysmorphism associated with MFS, shifting from the traditional impressive clinical view to a quantitative 3D not invasive evaluation of the facial features. Future research may expand the analysis on more patients and a wider set of facial measurements in order to facilitate the early diagnosis of the disorder. 


\section{References}

[1] OMIM, http://omim.org/entry/154700, accessed on 29 September 2016.

[2] Orphanet, http://www.orpha.net, accessed on 29 September 2016.

[3] CJ. Ramachandra et al., "Molecular pathogenesis of Marfan syndrome", in International Journal of Cardiology, Vol. 187, 2015, pp.585-591, http://dx.doi.org/10.1016/j.ijcard.2015.03.423.

[4] RM. Radke and H. Baumgartner, "Diagnosis and treatment of Marfan syndrome: an update" in Heart, Vol. 100, 2014, pp. 1382-1391, http://dx.doi.org/10.1136/heartjnl-2013-304709.

[5] BL. Loeys et al., "The revised Ghent nosology for the Marfan syndrome", in Journal of Medical Genetics, Vol. 47, 2010, pp. 476-485, http://dx.doi.org/10.1136/jmg.2009.072785.

[6] S. Sheikhzadeh et al., "A simple clinical model to estimate the probability of Marfan syndrome" in QJM: monthly journal of the Association of Physicians, Vol. 105, 2012, pp. 527-535, http://dx.doi.org/10.1093/ajmed/hcs008.

[7] PA. Cistulli et al., "Relationship between craniofacial abnormalities and sleep-disordered breathing in Marfan's syndrome", in Chest, Vol. 120, 2001, pp. 1455-1460.

[8] P. De Coster et al., "Craniofacial structure in Marfan syndrome: a cefalometric study", in American Journal of Medical Genetics Part A, Vol. 131, 2004, pp. 240-248, http://dx.doi.org/10.1002/ajmg.a.30393.

[9] R. Docimo et al., "Association between oro-facial defects and systemic alterations in children affected by Marfan syndrome", in Journal of Clinical and Diagnostic Research, Vol. 7, 2013, pp. 700-703, http://dx.doi.org/10.7860/JCDR/2013/5656.2885.

[10] JE. Bilodeau, "Retreatment of a patient with Marfan syndrome and severe root resorption", in American Journal of Orthodontics and Dentofacial Orthopedics, Vol. 137, 2010, pp. 123-134, http://dx.doi.org/10.1016/i.ajodo.2007.05.029.

[11] P. De Coster et al., "Oral manifestations of patients with Marfan syndrome: a case-control study", in Oral Surgery Oral Medicine Oral Pathology, Vol. 93, 2002, pp. 564-572, http://dx.doi.org/10.1067/moe.2002.121430.

[12] RH. Khonsari et al., "Extreme oral manifestations in a Marfan-type syndrome", in International Journal of Oral \& Maxillofacial Surgery, Vol. 39, 2010, pp. 622-625, http://dx.doi.org/10.1016/j.ijom.2010.01.006.

[13] T. Nishikawa et al., "Marfan's syndrome: Clinical manifestations in the oral-craniofacial area, biophysiological roles of fibrillins and elastic extracellular microfibers, and disease control of the fibrillin gene", in Journal of Oral and Maxillofacial Surgery, Medicine, and Pathology, Vol. 25, 2013 , pp. 374-388, http://dx.doi.org/10.1016/i.ajoms.2013.02.014.

[14] A. Utreja and CA. Evans, "Marfan syndrome-an orthodontic perspective", in Angle Orthodontist, Vol. 79, 2009, pp. 394-400, http://dx.doi.org/10.2319/112707-558.1.

[15] L. Westling et al., "Craniofacial manifestations in the Marfan syndrome: palatal dimensions and a comparative cephalometric analysis", in Journal of Craniofacial Genetics and Developmental Biology, Vol. 18, 1998, pp. 211-218.

[16] M. Kohler et al., "The prevalence of obstructive spleep apnoea and its association with aortic dilatation in Marfan's syndrome", in Thorax, Vol. 64, 2009, pp. 162-166, http://dx.doi.org/10.1136/thx.2008.102756.

[17] RK. da Palma et al., "Increased upper airway collapsibility in a mouse model of Marfan syndrome" in Respiratory Physiology \& Neurobiology, Vol. 207, 2015, pp. 58-60, http://dx.doi.org/10.1016/i.resp.2014.12.013.

[18] KJ. Lipscomb et al., "Evolving phenotype of Marfan's syndrome" in Archives of Disease in Childhood, Vol. 76, 1997, pp. 41-46.

[19] C. Sforza et al., "Three dimensional facial morphometry: from anthropometry to digital morphology", in Handbook of Anthropometry, Physical Measures of Human Form in Health and Disease. VR. Preedy (Ed), Springer Science+Business Media, LLC; Ch. 32, pp. 611-624, 2012; http://dx.doi.org/10.1007/978-1-4419-1788-1 32.

[20] K. Aldridge et al., "Precision and error of three-dimensional phenotypic measures acquired from 3dMD photogrammetric images", in American Journal of Medical Genetics Part A, Vol. 138, 2005, pp. 247-253, http://dx.doi.org/10.1002/ajmg.a.30959.

[21] C. Dolci et al., "3D craniofacial morphometric analysis of young subjects with Marfan syndrome: a preliminary report. In Proceedings of $6^{\text {th }}$ International Conference on 3D Body Scanning Technologies, Lugano, Switzerland, 2015. Pp. 54-60, http://dx.doi.org/10.15221/15.054.

[22] C. Sforza et al., "Soft- and hard-tissue facial anthropometry in three dimensions: what's new", in Journal of Anthropological Sciences, Vol. 91, 2013, pp. 59-84, http://dx.doi.org/10.4436/JASS.91007. 
[23] LG. Farkas (ed), Anthropometry of the head and neck, 2nd ed., Raven Press, New York, USA, 1994, pp. 427, http://dx.doi.org/10.1002/hed.2880170222.

[24] VF. Ferrario et al., "A three-dimensional study of sexual dimorphism in the human face", in International Journal of Adult Orthodontics \& Orthognathic Surgery, Vol. 9, 1994, pp. 303-310.

[25] VF. Ferrario et al.,"Growth and aging of facial soft tissues: a computerized three-dimensional mesh diagram analysis", in Clinical Anatomy, Vol. 16, 2003, pp. 420-433.

[26] C. Sforza et al., "Variation in facial soft tissues of Italian individuals with ectodermal dysplasia", in Cleft Palate Craniofacial Journal, Vol. 41, 2004, pp. 262-267, http://dx.doi.org/10.1597/03-033.1.

[27] C. Sforza et al., "Abnormal variations in the facial soft tissues of individuals with Down syndrome: Sudan versus Italy", in Cleft Palate Craniofacial Journal, Vol. 52, 2015, pp. 588-596, http://dx.doi.org/10.1597/14-082.

[28] C. Sforza et al., "Soft tissue facial morphometry in subjects with Moebius syndrome", in European Journal of Oral Sciences, Vol. 117, 2009, pp. 695-703, http://dx.doi.org/10.1016/.i.jom.2004.10.005.

[29] C. Sforza et al., "Three-dimensional facial distances of Northern Sudanese persons from childhood to young adulthood", in Journal of Cranio-Maxillo-Facial Surgery, Vol. 42, 2014, pp. e318-e326, http://dx.doi.org/10.1016/i.jcms.2013.10.013.

[30] M. de Menezes et al., "Evaluation of a 3D stereophotogrammetric method to identify and measure the palatal surface area in children with unilateral cleft lip and palate", in Cleft Palate Craniofacial Journal, Vol. 53, 2016, pp. 16-21, http://dx.doi.org/10.1597/14-076.

[31] M. de Menezes et al., "Accuracy and reproducibility of a 3-dimensional stereophotogrammetric imaging system", in Journal of Oral and Maxillofacial Surgery, Vol. 68, 2010, pp. 2129-2135, http://dx.doi.org/10.1016/i.joms.2009.09.036.

[32] C. Stheneur et al., "Study of phenotype evolution during childhood in Marfan syndrome to improve clinical recognition" in Genetics in Medicine, Vol. 16, 2014, pp. 246-250, http://dx.doi.org/10.1038/gim.2013.

[33] BL. Ting et al., "The diagnostic value of facial features of Marfan syndrome", in Journal of Children's Orthopaedics, Vol. 4, 2010, pp. 545-551, http://dx.doi.org/10.1007/s11832-010-0295-1. 